\title{
Distance Bounding in Noisy Environments
}

\author{
Dave Singelée and Bart Preneel \\ ESAT-COSIC, K.U.Leuven, \\ Kasteelpark Arenberg 10, 3001 Heverlee-Leuven, Belgium, \\ \{Dave.Singelee, Bart.Preneel\}@esat.kuleuven.be
}

\begin{abstract}
Location information can be used to enhance mutual entity authentication protocols in wireless ad-hoc networks. More specifically, distance bounding protocols have been introduced by Brands and Chaum at Eurocrypt'93 to preclude distance fraud and mafia fraud attacks, in which a local impersonator exploits a remote honest user. Hancke and Kuhn have proposed a solution to cope with noisy channels. This paper presents an improved distance bounding protocol for noisy channels that offers a substantial reduction (about 50\%) in the number of communication rounds compared to the Hancke and Kuhn protocol. The main idea is to use binary codes to correct bit errors occurring during the fast bit exchanges. Our protocol is perfectly suitable to be employed in low-cost, noisy wireless environments.
\end{abstract}

Keywords: Secure Localization, Distance Bounding, Wireless Sensor Networks, Entity Authentication.

\section{Introduction}

\subsection{Proximity based authentication}

In mobile networks, location information can be used to enhance mutual entity authentication protocols. Entities which are in a specific location or within a certain range of a device ("the verifier") are granted some privileges, in contrast to all other entities. In most scenarios, one would like to determine an upper bound on the distance to another entity. For instance, one could conduct a cryptographic identification protocol at the entrance to a building. Only entities with the correct credentials and who are not more than a few meters away are granted access to the building.

The concept of proximity based authentication is graphically depicted in Fig. 1. Authentication requests originating from devices that are located within the range $d$ of the verifier $V$ are accepted, all other requests are rejected. So in Fig. 1, authentication requests originating from device $A$ are accepted (and as a consequence, $A$ is granted some privileges), while the requests of $B$ are rejected. Contactless smart cards and RFID tokens are often used for proximity-based authentication (see Bardram et al. [1]). Such mobile devices have very limited processing power. Therefore, one has to employ low-cost cryptographic primitives to authenticate the mobile devices, and verify the distance between both parties. 


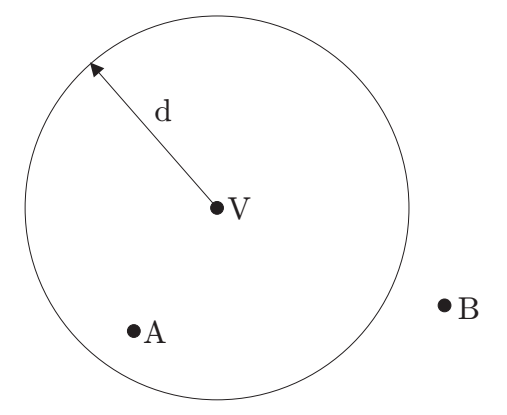

Fig. 1. Proximity based authentication

How can one securely verify if a certain device is within a specific range? There are several methods to accomplish this; one of them is to apply distance bounding protocols. These protocols enable the verifying party to determine an upper bound on the distance between itself and a prover, who claims to be within a certain range. Distance bounding protocols combine physical and cryptographic properties to determine an upper bound on the distance between verifier and prover. They allow the prover to authenticate itself to the verifier, and in the same time enable the verifying party to check if the prover is located within a certain range. Distance bounding techniques can measure the received signal strength (RSS) [2], the angle of arrival (AoA), or the time of flight (ToF) to estimate an upper bound on the distance. The first two techniques (RSS and AoA) are typically discarded because of security reasons: e.g., an attacker can construct a directional antenna to substantially increase the sending or receiving range [3]. This only leaves measuring the time of flight as a possible technique for secure distance bounding protocols.

\subsection{Organization of the paper}

This paper is organized as follows. In the introduction, we briefly discussed the idea of proximity based authentication in mobile ad-hoc networks. We put forward the idea of employing distance bounding protocols. The general principles of these protocols are discussed more in detail in Sect. 2. Section 3 and 4 describe two important distance bounding protocols: the protocol of Brands and Chaum protocol, and the protocol of Hancke and Kuhn protocol respectively. In Sect. 5, we show how to adapt the Mutual Authentication with Distance Bounding (MAD) protocol of Capkun et al. (extended version of the Brands and Chaum protocol) to make it noise resilient. This protocol requires about half of the number of communication rounds compared to other noise resilient distance bounding protocols, as will be shown in Sect. 6. Section 7 concludes the paper. 


\section{Background}

\subsection{How do distance bounding protocols work?}

Secure distance bounding protocols measure the time of flight to determine an upper bound on the distance between prover and verifier. This measurement is typically performed during a challenge-response protocol, the main building block of the distance bounding protocol. During $n$ fast bit exchanges, the time between sending a challenge and receiving the response is measured. Multiplying the time of flight with the propagation speed of the communication medium gives the distance between prover and verifier.

One should however take into account some important details. It should be impossible for the prover to send the response before receiving the challenge [4]. This implies that the response should be dependent on the (random) challenge. A second remark is that a challenge-response protocol is not sufficient. After execution of this protocol, the verifier only knows that some party is close. But how does one know that this entity is the prover? This problem arises for example in the Echo protocol [5]. That is why the prover has to identify itself somewhere in the scheme (not necessarily in the challenge-response protocol itself). Finally, one should notice that the round trip time is not equal to the propagation time. It takes some time to compute and transmit the response. This processing delay should be as small as possible compared to the propagation time, because we are only interested in the latter. We consider two communication technologies: (ultra-)sound and electromagnetic signals.

Ultra-sound: (Ultra-)sound is interesting to measure distances because it is relatively slow. The processing delay can hence be neglected compared to the propagation time and the accuracy of the measurements is not very critical. An example of a protocol using this technique is described by Kindberg and Zhang [6]. There are however some security problems. (Ultra-)sound is not resistant to physically present attackers. Such an attacker can modify the

medium (e.g., sound travels faster through metal than through the air) or use wormholes (e.g., by retransmitting the signal using electromagnetic waves) to claim that he is closer than he really is. By delaying the response, he can also claim to be further away.

Electromagnetic signals: An active attacker can not use wormholes since the signals travel with the speed of light and nothing propagates faster. This means that an attacker can only claim to be further away than he really is (by delaying the response). There are however some practical issues. The verifier has to be able to measure the round trip time with very high precision. A small deviation of the time of flight has a strong influence on the estimated distance. A similar problem is estimating the processing delay. One has to design the distance bounding protocol in such a way that the processing delay can be neglected to the (very small) time of flight. 


\section{$2.2 \quad$ Attack scenarios}

By employing the principle of distance bounding attacks in a clever way, one can preclude one or more fundamental attacks.

One wants to prevent a dishonest prover claiming to be closer than he really is. This attack is called distance fraud attack. It is relatively easy to design a distance bounding protocol which prevents this type of attack. Bussard presents in [7] an overview of location mechanisms that are resistant or partially resistant to distance fraud attacks.

Mafia fraud attacks, also called relay attacks, were first described by Desmedt [8]. In this attack scenario, both prover and verifier are honest, but a malicious intruder is performing the fraud. It is a man-in-the-middle attack where the intruder $I$ is modeled as a malicious prover $\bar{P}$ and verifier $\bar{V}$ that cooperate, as shown in Fig. 2. The malicious verifier $\bar{V}$ interacts with the honest prover $P$ and the malicious prover $\bar{P}$ interacts with the honest verifier $V$. The physical distance between the intruder and the verifier is small. This attack enables the intruder to identify itself to $V$ as $P$ being close to $V$, without any of $P$ and $V$ noticing the attack.

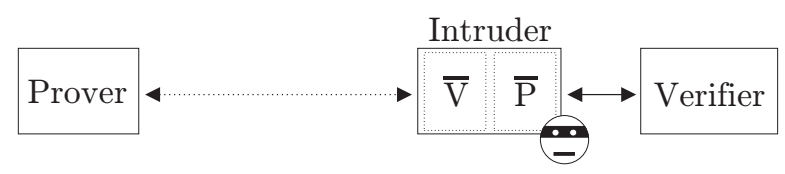

Fig. 2. Mafia fraud attack

Terrorist fraud attacks [8] are an interesting extension of the mafia fraud attack. The intruder and the prover will collaborate in this attack. This implies that a protocol which is resistant to terrorist fraud attacks, also prevents mafia fraud attacks. The terrorist fraud attack is shown in Fig. 3. The dishonest prover uses the intruder to convince the honest verifier that he is close, while in fact he is located at a large distance. The intruder does not know the private or secret key. This certainly has to be emphasized: if the intruder would know this private key, then it is impossible to make a distinction between the intruder and the prover, since distance bounding protocols only check if a party which knows the private key is close to the verifier. Several distance bounding protocols are resistant to terrorist fraud attacks $[7,9,10]$.

\subsection{Design principles for secure distance bounding protocols}

Without going in too many details, one can formulate the following (simplified) cryptographic design principles of distance bounding protocols:

- In at least one of the messages of the distance bounding protocol, the prover has to "identify" itself (e.g., by proving knowledge of a shared secret key). 


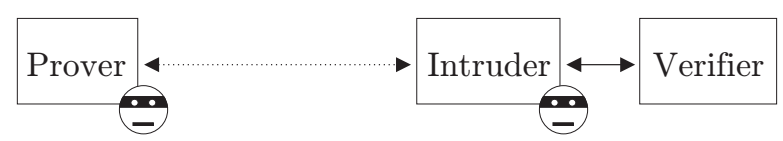

Fig. 3. Terrorist fraud attack

- To prevent mafia fraud attacks, the distance bounding protocol should contain a challenge-response protocol that consists of a series of rapid bit exchanges ( $n$ rounds in total) [4]. By measuring the round trip time in each of the $n$ rounds, the verifier can determine an upper bound on the distance between verifier and prover. To prevent the prover sending the response too soon, the challenge has to be random and unpredictable, and the response has to depend on this challenge.

- To avoid terrorist fraud attacks, one has to make sure that the fast bit exchanges and the phase in which the prover identifies itself, are intermingled in a cryptographic way. It has to be impossible to split the distance bounding protocol into these two distinct phases. There are at least two ways to accomplish this: either one uses the private (or symmetric) key during the fast bit exchanges, or one uses trusted hardware. For more details, we refer to $[9]$.

The design principles described above are not sufficient. Clulow et al. show that one has to optimize the choice of communication medium and transmission format according to the following four principles [11], if one wants to prevent certain "physical" attacks:

- Use a communication medium with a propagation speed as close as possible to the physical limit for propagating information through space-time.

- Use a communication format in which the recipient can instantly react on each single transmitted bit.

- Minimize the length of the symbol used to represent this single bit.

- Design the distance bounding protocol to cope with bit errors taking place during the rapid bit exchanges.

\section{The Distance Bounding Protocol of Brands and Chaum}

In 1993, Brands and Chaum presented their distance bounding protocol [4]. This clever protocol prevents mafia fraud attacks and embodies a series of $n$ rounds ( $n$ is a security parameter). Each round consists of a single bit challenge and a rapid single bit response. The delay time for receiving the responses enables the verifier to compute an upper bound on the distance. After correct execution of the distance bounding protocol, the verifier knows that an entity in possession of a certain secret is in the vicinity.

The protocol is carried out as follows. It contains three phases. First, the prover sends out a commitment to $n$ random bits $m_{i}$. Next, a series of $n$ fast bit 
exchanges is performed. The verifier sends a random challenge $\alpha_{i}$ to the prover. This challenge is XOR'ed with the bit $m_{i}$ and the result $\left(\beta_{i}\right)$ is sent back to the verifier. After the $n$ fast bit exchanges, the prover opens the commitment and signs the string $y$, which embodies the concatenation of the challenges $\alpha_{i}$ and the responses $\beta_{i}$. If the signature is correct, the protocol is successful. In each of the $n$ rounds, an attacker has a probability of $\frac{1}{2}$ to send a correct response [4]. Note that in every of the $n$ rounds, the prover has to compute the XOR of two bits. This can be done very efficiently in hardware.

Capkun et al. extended the protocol to MAD, a mutual authentication protocol using distance bounding [12]. This protocol has the advantage that both parties can estimate an upper bound on the distance between themselves, and learn each other's identity, which is not the case in the original protocol of Brands and Chaum. From all other points of view, both protocols are very similar. In the rest of this paper, we will assume that mutual entity authentication is required, and use the MAD protocol.

\section{The RFID Protocol of Hancke and Kuhn}

Both the distance bounding protocol of Brands and Chaum [4] and the MAD protocol [12] were not designed to cope with bit errors during the fast bit exchanges. A single bit error causes the protocol to fail. This can be an important problem in noisy environments such RFID. That is why Hancke and Kuhn proposed a distance bounding protocol [13] that can easily be extended to deal with bit errors.

The protocol is carried out as follows. First, prover and verifier exchange a random nonce ( $N_{P}$ and $N_{V}$ respectively). Both parties then use a pseudorandom function (typically a MAC algorithm such as CBC-MAC and HMAC is used since these have been shown to be pseudo-random functions [14]) to compute two $n$ bit sequences $v^{(0)}$ and $v^{(1)}$ (more in detail: $\left.M A C_{K}\left(N_{V}, N_{P}\right)=v^{(0)} \mid v^{(1)}\right)$. Next, a series of $n$ fast bit exchanges is performed. In each round, the verifier sends a random single bit challenge $C_{i}$ to the prover. If this challenge equals 0 , then the prover responds with the $i$-th bit of $v^{(0)}$. If the challenge equals 1 , then the prover sends the $i$-th bit of $v^{(1)}$. If all responses are correct, the protocol succeeds. In each round, an attacker has a probability of $\frac{3}{4}$ to send a correct response. After correct execution of the distance bounding protocol, the verifier knows that with probability $1-\left(\frac{3}{4}\right)^{n}$ an entity in possession of the secret key $K$ is in the vicinity.

If we compare the Hancke and Kuhn distance bounding protocol with the Brands and Chaum protocol, we notice that the latter requires a signature to be sent at the end of the protocol, while the former stops after the execution of the $n$ fast bit exchanges. So the Brands and Chaum protocol requires more bits to be interchanged on the slower communication channel, while the Hancke and Kuhn protocol needs more rounds of rapid single-bit exchanges. Munilla et al. proposed to use "void challenges" in the Hancke and Kuhn protocol [15] 
to improve the security. However the disadvantage of their solution is that is requires three (physical) states: 0,1 and void.

The Hancke and Kuhn protocol can easily be adapted to make it noise resilient. First one has to select a security parameter $x$. This parameter denotes the number of bit errors that are allowed during the $n$ fast bit exchanges; it depends on the bit error rate. The distance bounding protocol succeeds if at least $(n-x)$ of the responses sent by the prover are correct. The security parameter $x$ has to be chosen very carefully. Incrementing the number of allowed errors $x$ increases the false acceptance ratio dramatically. A more detailed discussion on the influence of the different security parameters will be presented in Sect. 6 .

\section{Noise Resilient Mutual Authentication with Distance Bounding}

As discussed in Sect. 3, the MAD protocol of Čapkun et al. has the nice property that in each of the $n$ rounds of the fast bit exchanges, an attacker only has a $\frac{1}{2}$ probability of replying to the verifier with a correct response. It also offers mutual entity authentication. On the other hand, the distance bounding protocol of Hancke and Kuhn can be easily made resilient to bit errors during the fast bit exchanges, which is a very desirable feature. It would be ideal to combine the good properties of both distance bounding protocols.

A trivial way of making the MAD protocol noise resilient, is exchanging all challenges and responses again on a slower communication channel with error correction (of course, this has to be done after the fast bit exchanges). However, this is not very efficient. We will now present an efficient modification of the MAD protocol, which is also resilient to some bit errors (we allow $x$ bit errors in total) during the fast bit exchanges. Our protocol, in which the two parties (denoted by Alice and Bob) will authenticate each other, is shown in Fig. 4.

The protocol is carried out as follows. First, both parties agree on an $(n, k)$ Error Correcting Code (ECC). In order to correct at least $x$ bit errors during the fast bit exchanges, this binary code should have a minimal Hamming distance $d_{\min }$ such that $x=\left\lfloor\frac{d_{\min }-1}{2}\right\rfloor$. More information on which $(n, k)$ error correcting code to use for a given distance $d_{\min }$ can be found in [16-19]. Note that we consider both linear and non-linear codes.

Next, Alice and Bob generate $k$ random bits $\left(r_{1}, \ldots, r_{k}\right.$ and $s_{1}, \ldots, s_{k}$ respectively). These $k$ bits are extended to $n$-bit strings $\left(r_{1}, \ldots, r_{n}\right.$ and $\left.s_{1}, \ldots, s_{n}\right)$ by applying the error correcting code described above and a commitment to this string is sent to the other party. Several secure commitment schemes can be used in our distance bounding protocol. E.g., one could first generate a 128-bit random string, then concatenate it with the $n$-bit string $r_{1}, \ldots, r_{n}$ or $s_{1}, \ldots, s_{n}$ and apply a cryptographic hash function to the resulting string of bitlength $128+n$. The output of this function is sent to the other party. To open the commitment, one should reveal the 128-bit random string. This commitment scheme is unconditionally hiding and conditionally binding. More information can be found in $[20]$. 


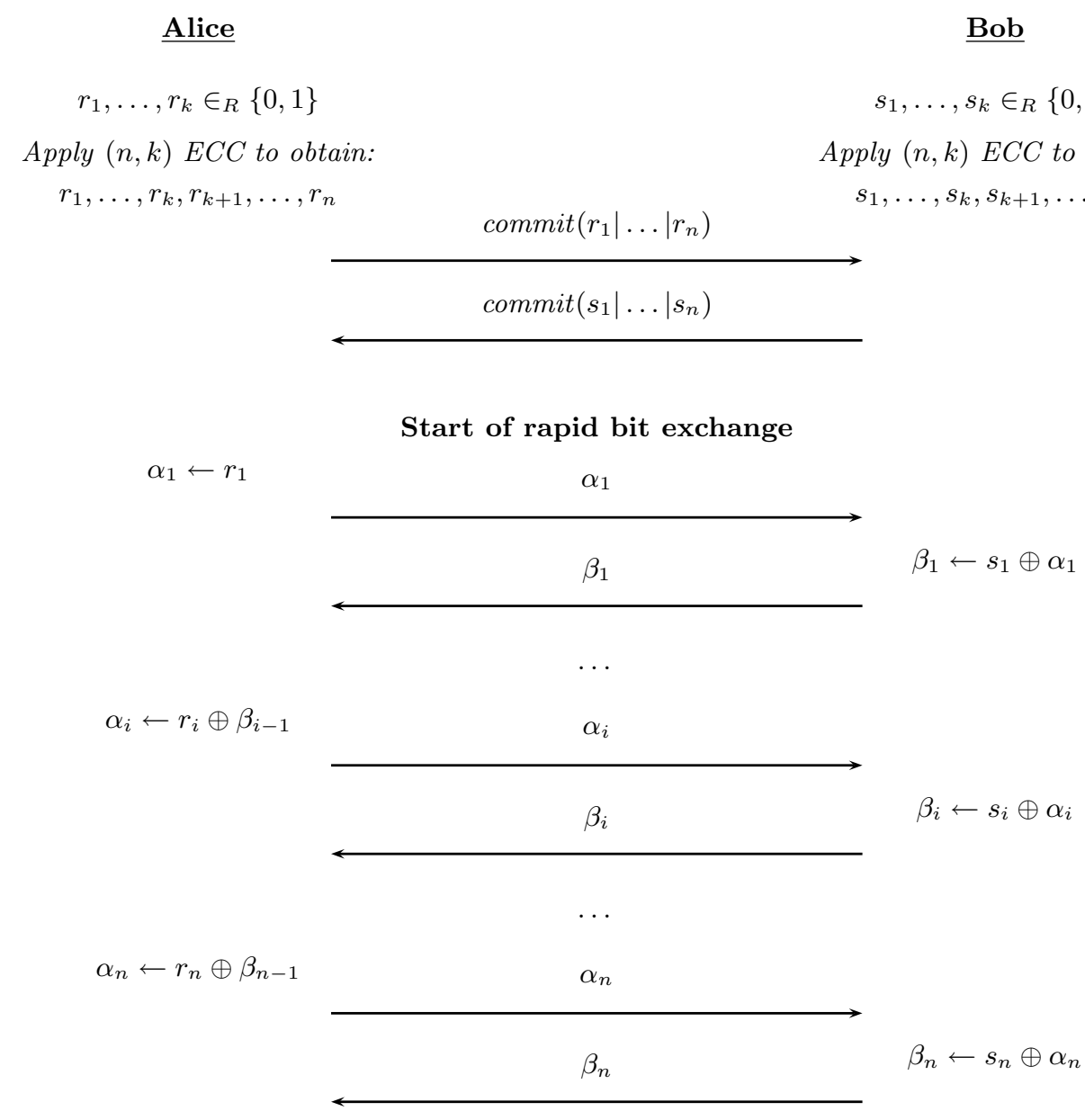

End of rapid bit exchange

\begin{tabular}{|c|c|c|}
\hline$s_{i} \leftarrow \alpha_{i} \oplus \beta_{i}$ & & $r_{1} \leftarrow \alpha_{1}$ and $r_{i} \leftarrow \alpha_{i} \oplus \beta_{i-1}$ \\
\hline Use ECC to correct errors: & & Use ECC to correct errors: \\
\hline$\Rightarrow s_{1}, \ldots, s_{k}$ & & $\Rightarrow r_{1}, \ldots, r_{k}$ \\
\hline$y_{A} \leftarrow M A C_{K}\left(r_{1}\left|s_{1}\right| \ldots\left|r_{k}\right| s_{k}\right)$ & (open commit), $y_{A}$ & $y_{B} \leftarrow M A C_{K}\left(s_{1}\left|r_{1}\right| \ldots\left|s_{k}\right| r_{k}\right)$ \\
\hline & (open commit),$y_{B}$ & \\
\hline Verify commit & & Verify commit \\
\hline Verify $y_{B}$ & & Verify $y_{A}$ \\
\hline
\end{tabular}

Fig. 4. Noise resilient mutual entity authentication with distance bounding protocol 
During the $n$ fast bit exchanges, the following two steps are repeated $n$ times:

- Alice sends the bit $\alpha_{i}$ to Bob where $\alpha_{1}=r_{1}$ and $\alpha_{i}=r_{i} \oplus \beta_{i-1}$.

- Bob sends the bit $\beta_{i}$ to Alice where $\beta_{i}=s_{i} \oplus \alpha_{i}$.

In each round, the time between sending $\alpha_{i}$ and receiving $\beta_{i}$ (or sending $\beta_{i}$ and receiving $\alpha_{i+1}$ ) is measured. The maximum round trip time is selected and this measurement determines an upper bound on the estimation of the distance between Alice and Bob. After the fast bit exchanges, both parties use the $(n, k)$ ECC to correct bit errors (each party can correct a maximum of $x$ bit failures) and this way recover the bits $s_{1}, \ldots, s_{k}$ and $r_{1}, \ldots, r_{k}$ respectively. Finally, Alice (and Bob) compute a $M A C$ on the concatenation of $r_{i}$ and $s_{i}$ (or $s_{i}$ and $r_{i}$ ) and open the commitment sent in the beginning of the protocol. If the MAC and the commitment are correct, the protocol is successful. In each of the first $k$ rounds, an attacker has a $\frac{1}{2}$ probability of sending a correct response. Note that our protocol only requires low-cost cryptographic primitives, and hence is perfectly suitable to be employed in resource constrained wireless networks.

\section{Performance Analysis}

\subsection{False rejection and false acceptance ratio}

In our analysis, we assume that the fast communication channel used during the rapid bit exchanges is symmetric. So a bit error is as likely to occur in a challenge as in a response. We also assume that a bit error is independent of previous bit errors. The bit error rate is denoted by $P_{b}$.

Before numerically analyzing and deriving the statistical properties of our distance bounding protocol, let us first clearly define the notion of a round (during the fast bit exchanges). This definition depends on the distance bounding protocol that is being used. In the Hancke and Kuhn protocol, we define a round as a challenge and the corresponding response. In our noise resilient MAD protocol, a round are two consecutive messages (so $\alpha_{i}$ and $\beta_{i}$, or $\beta_{i}$ and $\alpha_{i+1}$ ).

Some of the challenges and/or responses will be corrupted by noise. The probability that a round fails is denoted by $\varepsilon$. A round fails if the verifying party receives an incorrect response, or if one of the parties in our noise resilient MAD protocol gets a corrupted bit $\overline{r_{i}}$ or $\overline{s_{i}}$. Let us first have a look to the Hancke and Kuhn protocol. A bit error can appear in the challenge, or in the response (both with probability $P_{b}$ ). We neglect the probability that a bit error occurs in both messages. If the prover receives an incorrect challenge, he still has a $\frac{1}{2}$ probability of sending the correct response (this event happens when the responses for both the challenges 0 and 1 are equal). If the verifier receives a corrupted response, the round fails certainly. So one can easily compute the probability $\varepsilon_{H}$ that a round fails in the Hancke and Kuhn distance bounding protocol:

$$
\varepsilon_{H}=\frac{3}{2} P_{b}
$$


In our noise resilient MAD protocol, a round fails by definition with $100 \%$ probability when a bit $\alpha_{i}$ or $\beta_{i}$ is corrupted. The probability $\varepsilon_{M A D}$ that a round fails in our noise resilient MAD protocol is equal to

$$
\varepsilon_{M A D}=2 P_{b} .
$$

We can now compute the false rejection and false acceptance ratio, two important parameters to evaluate (noise resilient) distance bounding protocols. An honest prover is falsely rejected if more than $x$ bit errors occur during the fast bit exchanges (which consist out of $n$ rounds). The false rejection ratio depends on the probability $\varepsilon$ (equal to $\varepsilon_{H}$ or $\varepsilon_{M A D}$ ) and is equal to

$$
P_{F R}=\sum_{i=0}^{n-x-1}\left(\begin{array}{l}
n \\
i
\end{array}\right) \cdot(1-\varepsilon)^{i} \cdot \varepsilon^{(n-i)} .
$$

This expression is valid for both distance bounding protocols.

An attacker can use the uncertainty of which bits are corrupted by noise, to its advantage. In the worst case, no bit errors occur, but the (honest) verifier expects a maximum of $x$ bit errors. As a consequence, an attacker only has to guess $(n-x)$ responses right in the Hancke and Kuhn distance bounding protocol to perform a successful attack (without taking into account noise, an attacker should have to guess all $n$ responses correctly to be successful). The false acceptance ratio of the Hancke and Kuhn protocol equals

$$
P_{F A}=\sum_{i=n-x}^{n}\left(\begin{array}{c}
n \\
i
\end{array}\right) \cdot\left(\frac{3}{4}\right)^{i} \cdot\left(\frac{1}{4}\right)^{(n-i)} .
$$

The situation is slightly different in our noise resilient MAD protocol. Since the first $k$ bits of $r_{i}$ and $s_{i}$ are independent and uniformly distributed in $\{0,1\}$, the two sequences $\alpha_{i}$ and $\beta_{i}$ are independent up to the point where the index is $k$ (and by consequence, the first $k$ rounds of rapid single-bit exchanges are also independent). If the commitments sent in the beginning of the protocol are (un)conditionally hiding and binding, it is infeasible for a computationally bounded attacker to determine these bits in advance. The last $(n-k)$ bits of $r_{i}$ and $s_{i}$ depend of the first $k$ bits and can be easily computed by applying the $(n, k)$ error correcting code. In the worst case scenario (no bit errors occur), the last $(n-k)$ bits of the sequences $\alpha_{i}$ and $\beta_{i}$ can be computed in advance (from the moment the first $k$ rounds are conducted) and do not offer extra security. To be successful, an attacker hence has to correctly guess the first $k$ bits $r_{i}$ (or $\left.s_{i}\right) .{ }^{1}$ The false acceptance ratio of our noise resilient MAD protocol equals

$$
P_{F A}=\left(\frac{1}{2}\right)^{k}
$$

\footnotetext{
${ }^{1}$ Note that the number of allowed errors $x$ is always strictly smaller than the minimal Hamming distance $d_{m i n}$ of the $(n, k)$ error correcting code.
} 


\subsection{Numerical results}

Both noise resilient distance bounding protocols have some interesting characteristics. We will now compare both protocols, and have a closer look at the most interesting properties.

An attacker has a major advantage when bit errors due to noise can appear. In the worst case scenario, an honest verifier expects to receive some corrupted bits due to noise, while in fact there is no noise at all. As a direct consequence, an attacker can obtain a major advantage. Whenever he guesses a response wrongly, he can blame it to the noise. As long as an attacker has a maximum of $x$ wrong guesses, the Hancke and Kuhn distance bounding protocol will be successful (because the verifier believes that the incorrect bits were corrupted by noise). The more errors that are allowed, the larger the false acceptance ratio. The same property is also valid for our noise-resilient MAD protocol. For a fixed number $n$ of rounds, the more errors $x$ have to be corrected, the smaller the parameter $k$ has to be [16-19]. And because only the first $k$ rounds of the fast bit exchanges contribute to the security, the false acceptance ratio will increase with decreasing $k$. This property is demonstrated for both distance bounding protocols in Table 1. In this numerical example, $n=37$ and the bit error rate $P_{b}$ is 0.01 . The error correcting codes for our noise resilient MAD protocol have been selected following [16]. The results in Table 1 clearly show that the false acceptance ratio increases significantly with the number $x$ of allowed errors. One can also notice that the false acceptance ratio is remarkably smaller in our noise resilient MAD protocol (several orders of magnitude). We will discuss this observation later in this section.

Table 1. Influence of the number of allowed errors $x$ on the false acceptance ratio $P_{F A}$ for $n=37$ and $P_{b}=0.01$

\begin{tabular}{|c|c|c|c|}
\hline \# allowed errors & Hancke-Kuhn & \multicolumn{2}{|c|}{ Noise Resilient MAD } \\
\cline { 3 - 4 }$x$ & $P_{F A}$ & $(n, k)$ ECC & $P_{F A}$ \\
\hline 4 & 0.0284 & $(37,16)$ & $1.5259 \cdot 10^{-5}$ \\
3 & 0.0089 & $(37,22)$ & $2.3842 \cdot 10^{-7}$ \\
2 & 0.0021 & $(37,26)$ & $1.4901 \cdot 10^{-8}$ \\
1 & $3.1784 \cdot 10^{-4}$ & $(37,31)$ & $4.6566 \cdot 10^{-10}$ \\
0 & $2.3838 \cdot 10^{-5}$ & $(37,37)$ & $7.2760 \cdot 10^{-12}$ \\
\hline
\end{tabular}

The false rejection ratio is slightly lower in the Hancke and Kuhn distance bounding protocol. Whereas noise helps an attacker to deceive an honest verifier, it is disadvantageous for an honest prover behaving correctly. The higher the bit error rate $P_{b}$, the higher the probability that the distance bounding 
protocol will fail because of too many bit errors during the fast bit exchanges. If no bit errors occur during the fast bit exchange phase, an honest prover will always be able to authenticate itself successfully. To decrease the false rejection ratio, one has to allow more bit errors to take place (denoted by $x$ ) for a fixed number $n$ of rounds, or decrease the number of rounds (without changing $x$ ). The choice of the parameter $x$ has to be in accordance to the expected number of errors, which depends on the number $n$ of rounds and the bit error rate $P_{b}$.

As demonstrated in (1) and (2) in Sect. 6.1, the probability $\varepsilon_{M A D}$ of a round to fail in our noise resilient MAD protocol is higher than in the Hancke and Kuhn distance bounding protocol $\left(\varepsilon_{H}\right)$. A direct consequence of this fact, is that the false rejection ratio is lower in the Hancke and Kuhn protocol (for equal number $n$ of rounds and allowed errors $x$ ). This property is demonstrated in Table 2 . In this numerical example, $n=37$ and $P_{b}=0.01$. Note that the difference in false rejection ratio between both distance bounding protocols is relatively small. When the number $n$ of rounds is larger (e.g., around 50), one should allow one or two more errors to occur in our MAD resilient to keep the false rejection ratio comparable in both protocols (e.g., for $n=47$ : $P_{F R}($ Hancke, $x=9)=$ $\left.1.7985 \cdot 10^{-9} \approx P_{F R}(M A D, x=10)=1.8353 \cdot 10^{-9}\right)$.

Table 2. Comparison of the false rejection ratio for $n=37$ and $P_{b}=0.01$

\begin{tabular}{|c|c|c|}
\hline \# allowed errors $x$ & Hancke-Kuhn: $P_{F R}$ & Noise Res. MAD: $P_{F R}$ \\
\hline$x=6$ & $1.1849 \cdot 10^{-6}$ & $7.7770 \cdot 10^{-6}$ \\
\hline$x=5$ & $1.7760 \cdot 10^{-5}$ & $8.7314 \cdot 10^{-5}$ \\
\hline$x=4$ & $2.2184 \cdot 10^{-4}$ & $8.1806 \cdot 10^{-4}$ \\
\hline$x=3$ & 0.0023 & 0.0062 \\
\hline$x=2$ & 0.0179 & 0.0375 \\
\hline$x=1$ & 0.1062 & 0.1689 \\
\hline$x=0$ & 0.4283 & 0.5265 \\
\hline
\end{tabular}

The false acceptance ratio is significantly higher in the Hancke and Kuhn distance bounding protocol. As demonstrated above, to decrease the false acceptance ratio, one has to allow fewer bit errors (denoted by $x$ ) for a fixed number $n$ of rounds, or increase the number of rounds (without changing $x$ ).

Table 1 shows that the false acceptance ratio is remarkably higher in the Hancke and Kuhn protocol. The main reason is that an attacker has a $\frac{3}{4}$ probability of guessing a response correctly in the Hancke and Kuhn protocol, but only a $\frac{1}{2}$ probability in our noise resilient MAD protocol. This difference is amplified exponentially, and not entirely compensated by the fact that an attacker has to guess more bits correctly in the Hancke and Kuhn protocol $((n-x)$ bits, compared to $k$ bits in our noise resilient MAD protocol). This property is also demonstrated in Table 3. In this numerical example, $n=63$ and $P_{b}=0.02$. The error correcting codes have been selected based on [17] (some of these codes are non-linear). 
Table 3. Comparison of the false acceptance ratio for $n=63$ and $P_{b}=0.02$

\begin{tabular}{|c|c|c|c|}
\hline \# allowed errors & Hancke-Kuhn & \multicolumn{2}{|c|}{ Noise Resilient MAD } \\
\cline { 3 - 4 }$x$ & $P_{F A}$ & $(n, k)$ ECC & $P_{F A}$ \\
\hline 13 & 0.2611 & $(63,12)$ & $2.4414 \cdot 10^{-4}$ \\
10 & 0.0584 & $(63,18)$ & $3.8147 \cdot 10^{-6}$ \\
7 & 0.0052 & $(63,28)$ & $3.7253 \cdot 10^{-9}$ \\
5 & $5.1111 \cdot 10^{-4}$ & $(63,37)$ & $7.2760 \cdot 10^{-12}$ \\
3 & $2.3004 \cdot 10^{-5}$ & $(63,47)$ & $7.1054 \cdot 10^{-15}$ \\
1 & $2.9599 \cdot 10^{-7}$ & $(63,57)$ & $6.9389 \cdot 10^{-18}$ \\
\hline
\end{tabular}

Note that the difference in false acceptance ratio is quite large: even allowing a slightly lower number of errors $x$ in the Hancke and Kuhn protocol does not really help to remove this inequality (e.g., if we have a look at Table 3: $P_{F A}($ Hancke, $\left.x=1)=2.9599 \cdot 10^{-7}>P_{F A}(M A D, x=7)=3.7253 \cdot 10^{-9}\right)$. One could also fix the number of allowed errors $x$, but perform more fast bit exchanges in the Hancke and Kuhn protocol (or in other words, increase the number $n$ of rounds). This would however make the distance bounding protocol more expensive, as the cost is directly related to the number $n$ of fast bit exchanges. Figure 5 shows the relation between the false acceptance ratio and the number of rounds $n$, for a fixed number of allowed errors $x$. In this example, we fixed the number $x$ of allowed errors to 5 , the bit error rate $P_{b}$ is 0.005 , and the information on which error correcting code to use (in our noise resilient MAD protocol) is based on [17]. Figure 5 demonstrates that the Hancke and Kuhn protocol needs about twice as many rounds $n$ to obtain the same false acceptance ratio. This largely increases the cost, and also causes the false rejection ratio to rise several orders of magnitude. If mutual autentication is required, the number of fast bit exchanges $n$ even needs to be doubled (and becomes the quadruple of the number of rounds needed in our noise resilient MAD protocol).

\section{Conclusion}

Location information can be used to enhance mutual entity authentication protocols in wireless ad-hoc networks. Distance bounding protocols, which have been introduced by Brands and Chaum at Eurocrypt'93 to preclude distance fraud and mafia fraud attacks, can be employed in proximity based authentication schemes to determine an upper bound on the distance to another entity. Hancke and Kuhn have presented a solution to cope with noisy channels, which is important in mobile environments.

In this paper, we have extended the mutual authentication distance bounding (MAD) protocol of Capkun et al. to make it tolerant to bit errors. This is accomplished by employing binary codes to correct bit errors occurring during the fast bit exchanges, the main building block of the distance bounding protocol. The protocol is best used for radio frequency communications, which is 


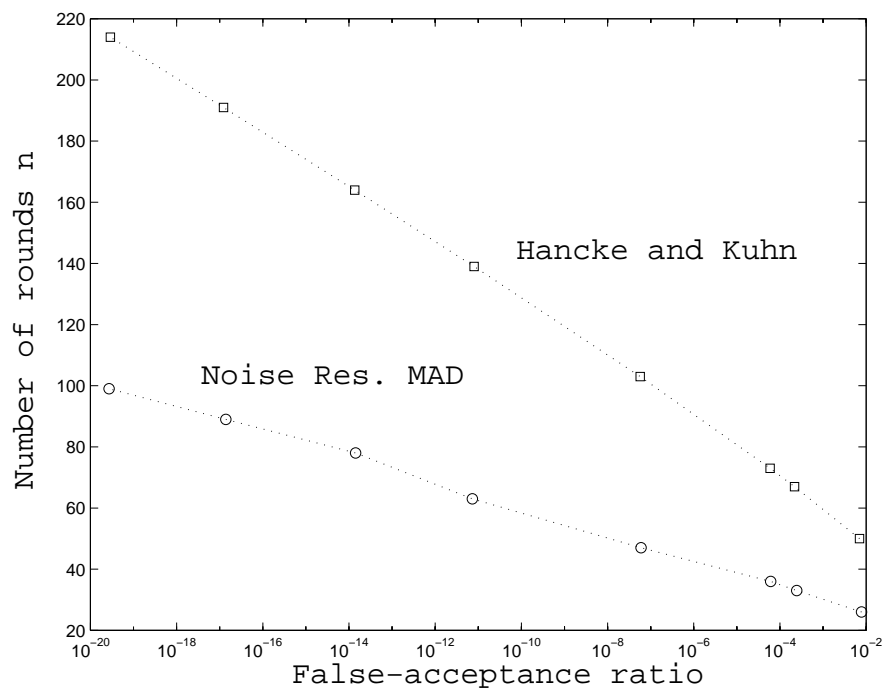

Fig. 5. Relation between the number $n$ of rounds and the false acceptance ratio $P_{F A}$ for $x=5$ and $P_{b}=0.005$

more suited for secure applications than ultrasonic. Our noise resilient MAD protocol requires about half of the number of communication rounds to obtain the same false acceptance ratio as the Hancke and Kuhn protocol. It also provides mutual entity authentication and can be made robust to terrorist fraud attacks by executing the protocol in trusted hardware. Compared to the Hancke and Kuhn protocol, our noise resilient MAD protocol requires slightly more bits to be exchanged on the slower communication channel. The exact total cost depends on the technical characteristics of the communication medium and the required level of security. Our distance bounding protocol is perfectly suitable to be employed in low-cost, noisy wireless environments.

Acknowledgments. Dave Singelée is funded by a research grant of the Katholieke Universiteit Leuven. This work was supported in part by the Concerted Research Action (GOA) Ambiorics 2005/11 of the Flemish Government and by the IAP Programme P6/26 BCRYPT of the Belgian State (Belgian Science Policy). The authors would like to thank Markus G. Kuhn for the valuable comments and interesting discussions on distance bounding protocols.

\section{References}

1. Bardram, J., Kjær, R., Pedersen, M.: Context-Aware User Authentication - Supporting Proximity-Based Login in Pervasive Computing. In: Proceedings of the 5th International Conference on Ubiquitous Computing (Ubicomp '03). Lecture Notes in Computer Science, LNCS 2864, Springer-Verlag (2003) 107-123 
2. Bahl, P., Padmanabhan, V.: RADAR: An In-Building RF-based User Location and Tracking System. In: Proceedings of the 19th annual conference on Computer Communications (INFOCOM '00). Volume 2., IEEE (2000) 775-784

3. Cheung, H.: The Bluesniper Rifle. http://www.tomsnetworking.com/ Sections-article106.php (2004)

4. Brands, S., Chaum, D.: Distance-Bounding Protocols. In: Advances in Cryptology - EUROCRYPT '93. Lecture Notes in Computer Science, LNCS 765, SpringerVerlag (1994) 344-359

5. Sastry, N., Shankar, U., Wagner, D.: Secure Verification of Location Claims. http: //www.cs.berkeley.edu/ nks/locprove/csd-03-1245.pdf (2003)

6. Kindberg, T., Zhang, K.: Validating and Securing Spontaneous Associations between Wireless Devices. In: Proceedings of the 6th Information Security Conference (ISC '03). Lecture Notes in Computer Science, LNCS 2851, Springer-Verlag (2003) $44-53$

7. Bussard, L.: Trust Establishment Protocols for Communicating Devices. PhD thesis, ENST Paris (2004) 233 pages.

8. Desmedt, Y.: Major Security Problems with the "Unforgeable" (Feige)-Fiat-Shamir Proofs of Identity and how to overcome them. In: Proceedings of SecuriCom ' 88. (1988) 15-17

9. Singelée, D., Preneel, B.: Location Verification using Secure Distance Bounding Protocols. In: Proceedings of the 2nd IEEE International Conference on Mobile, Ad Hoc and Sensor Systems (MASS '05). (2005) 834-840

10. Waters, B., Felten, E.: Proving the Location of Tamper-Resistant Devices. http: //www.cs.princeton.edu/bwaters/research/location_proving.ps (2003)

11. Clulow, J., Hancke, G., Kuhn, M., Moore, T.: So Near and Yet So Far: DistanceBounding Attacks in Wireless Networks. In: Proceedings of the 3rd European Workshop on Security and Privacy in Ad-Hoc and Sensor Networks (ESAS '06). Lecture Notes in Computer Science, LNCS 4357, Springer-Verlag (2006) 83-97

12. Čapkun, S., Buttyán, L., Hubaux, J.: SECTOR: Secure Tracking of Node Encounters in Multi-hop Wireless Networks. In: Proceedings of the 1st ACM Workshop on Security of Ad Hoc and Sensor Networks (SASN '03). (2003) 21-32

13. Hancke, G., Kuhn, M.: An RFID Distance Bounding Protocol. In: Proceedings of the 1st International Conference on Security and Privacy for Emerging Areas in Communications Networks (SECURECOMM '05), IEEE Computer Society (2005) $67-73$

14. Menezes, A.J., van Oorschot, P.C., Vanstone, S.A.: Handbook of Applied Cryptography. CRC Press (1996)

15. Munilla, J., Ortiz, A., Peinado, A.: Distance Bounding Protocols with voidchallenges for RFID. Workshop on RFID Security - RFIDSec '06 (2006)

16. Jaffe, D.: Information about binary linear codes. http://www.math.unl.edu/ ijaffe2/codes/webcodes/codeform.html

17. Litsyn, S.: Table of Nonlinear Binary Codes. http://www.eng.tau.ac.il/ litsyn/tableand/index.html

18. MacWilliams, F., Sloane, N.: The Theory of Error-Correcting Codes. NorthHolland (1977)

19. Pless, V., Brualdi, R., Huffman, W.: Handbook of Coding Theory. Elsevier Science Inc. (1998)

20. Damgård, I.: Commitment Schemes and Zero-Knowledge Protocols. In: Lectures on Data Security: Modern Cryptology in Theory and Practice. Lecture Notes in Computer Science, LNCS 1561, Springer-Verlag (1999) 63-86 